\title{
STUDY OF CPM DEVICE USED FOR REHABILITATION AND EFFECTIVE PAIN MANAGEMENT FOLLOWING KNEE ALLOPLASTY
}

\author{
R. TROCHIMCZUK* \\ Bialystok University of Technology \\ Faculty of Mechanical Engineering \\ Department of Automatic Control and Robotics \\ Wiejska 45C, 15-351 Bialystok, POLAND \\ E-mail: r.trochimczuk@pb.edu.pl \\ T. KUŹMIEROWSKI \\ Lomza State University of Applied Sciences (LSUAS) \\ Institute of Informatics and Automation \\ Akademicka 14, 18-400 Lomza, POLAND \\ E-mail: tkuzmierowski@pwsip.edu.pl \\ P. ANCHIMIUK \\ Non-Public Health Care Institution Specialist Clinic „Rehabilitacja” \\ Słonimska 15/1, 15-028 Bialystok, POLAND
}

\begin{abstract}
This paper defines the design assumptions for the construction of an original demonstration of a CPM device, based on which a solid virtual model will be created in a CAD software environment. The overall dimensions and other input parameters for the design were determined for the entire patient population according to an anatomical atlas of human measures. The medical and physiotherapeutic community were also consulted with respect to the proposed engineering solutions. The virtual model of the CPM device that will be created will be used for computer simulations of changes in motion parameters as a function of time, accounting for loads and static states. The results obtained from computer simulation will be used to confirm the correctness of the design adopted assumptions and of the accepted structure of the CPM mechanism, and potentially to introduce necessary corrections. They will also provide a basis for the development of a control strategy for the laboratory prototype and for the selection of the strategy of the patient's rehabilitation in the future. This paper will be supplemented with identification of directions of further research.
\end{abstract}

Key words: CPM device, rehabilitation robot, effective pain management, knee alloplasty, FEM analysis.

\section{Introduction}

The human knee is the largest joint in the human body, anatomically complex yet mechanically simple. A set of ligaments provide additional stabilization during flexion and extension as well as a small degree of rotation. Pain and limitations of mobility as well as other damage to this joint resulting from trauma or disease (degenerative and rheumatic diseases, neoplasms) or congenital anomalies, make it impossible for patients to lead their daily professional and private lives to a significant extent. In certain cases, surgical intervention supported by various forms of post-operative physiotherapy (including various types of kinesiotherapy supported by physiotherapy) is necessary. One form of surgical intervention that is practiced is knee alloplasty. This surgical procedure is based on removal of damaged articular surfaces of the

\footnotetext{
* To whom correspondence should be addressed
} 
joint and replacing them with synthetic prostheses. The surgical procedure is performed so that the patient can recover joint mobility in the full range of motion required for daily locomotion. Alloplasty is one of the most effective methods of medical treatment in terms of its effect on the patient's functioning, relieving pain and generally improving the patient's quality of life. It is one of the most commonly performed procedures in adult orthopedics. According to [1], it is estimated that 55 thousand joint alloplasty procedures were performed in Poland in 2012. Other sources [2] state that the number of total knee alloplasties (TKA) and replacements (TKR) in the USA in that same year is estimated at 500 thousand, noting a growing trend. Pain in locomotor organs during the post-operative period may contribute to limitation of the mobility of the joint that was operated upon, extend hospitalization, and even deteriorate joint function after the completion of treatment. Thus, along with anti-inflammatory pharmacology, one of the measures used in post-operative rehabilitation and pain management is exercise (kinesiotherapy) supported by mechatronic devices that perform continuous passive motion (CPM) [3-5]. The goal of such treatment after total knee alloplasty is to restore mobility in the knee joint that was operated on to the greatest possible extent as well as full muscular control over it. This is linked to restoration of proper static and dynamic function of the joint and the entire lower limb. The healing of damaged tissue that takes place as a result of surgery is a multi-stage biological process in which an inflamed state is dominant. This imposes the necessity of adapting rehabilitation procedure to individual stages of the tissue healing process after surgery.

This paper defines the design assumptions for the construction of an original demonstration of a CPM device, based on which a solid virtual model will be created in a CAD - SolidWorks software environment. The overall dimensions and other input parameters for the design were determined for the entire patient population according to an anatomical atlas of human measures. The medical and physiotherapeutic community were also consulted with respect to the proposed engineering solutions. The virtual model of the CPM device that will be created will be used for computer simulations of changes in motion parameters as a function of time, accounting for loads and static states. The results obtained from computer simulation will be used to confirm the correctness of the design assumptions adopted and of the accepted structure of the CPM mechanism, and potentially to introduce necessary corrections. They will also provide a basis for the development of a control strategy for the laboratory prototype and for selection of the strategy of the patient's rehabilitation in the future. This paper will be supplemented with identification of directions of further research.

\section{Alloplasty - injunctions and used implants}

The general indication for alloplasty is pain and impairment of joint function as a consequence of illnesses and trauma. Detailed indications for a procedure of this type are, above all [1]:

- osteoarthritis;

- $\quad$ rheumatic diseases, e.g. rheumatoid arthritis;

- avascular necroses in the area of articular facets;

- $\quad$ post-traumatic deformations within a limb;

- joint damage during progression of fracture without healing prognosis;

- neoplasms of the locomotor system leading to articular resection.

Total knee arthroplasty is a basic surgical procedure in patients with extensive deformations of this joint. It is also applied in patients with degenerative diseases, rheumatoid arthritis, and it is considered to be the method of choice in such cases. The main objectives of the procedure are, above all [6]:

- relieving or significant reduction of pain;

- correction of the disrupted joint axis and improvement of its stability;

- restoration to the greatest possible extent of the correct range of movements as well as proper static and dynamic function of the joint;

- improvement of the patient's general quality of life. 
Prostheses with the lowest degree of constraint, covering the smallest possible part of the joint and adjoining bones, are used for knee arthroplasty procedures [6]. The following prosthesis solutions are applied depending on the area of joint replacement:

- total prostheses - consisting of a femoral and tibial component with a polyethylene insert;

- partial, unicompartmental prostheses - replace only one damaged part of the joint and usually consist of two components, analogously to the total prosthesis;

- $\quad$ prostheses of the femoropatellar joint - most frequently consist of one component, i.e., the femoral articular facet;

- partial, bicompartmental prostheses - replace the medial or lateral compartment and additionally the frontal compartment (femoropatellar joint);

- tricompartmental prostheses - encompass all compartments of the knee joint along with the femoropatellar joint.

Knee joint prostheses are classified as follows according to the degree of constraint between their components:

- non-constrained - these are one of the most frequently used knee joint prostheses, in which components are not constrained;

- semi-constrained - implanted in the case of insufficiency of one of the collateral ligaments, components are partially constrained;

- constrained prosthesis with rotation - applied in the case of total absence of the knee ligament system, enabling the patient to perform flexion, extension movement as well as rotation, with total constraint between components;

- constrained prostheses - only allow flexion/extension movements due to the fully constrained joint between components.

Regardless of the type of prosthesis applied, the patient undergoes rehabilitation treatment after the surgical procedure. This is intended to restore mobility in the knee that was operated onto the greatest possible extent as well as to restore full muscular control. This is directly linked to restoration of proper static and dynamic function of the joint and the entire lower limb. CPM devices are the most commonly used to support the patient in rehabilitation activities.

\section{Engineering aspects of the design of CPM machine}

When undertaking the task of designing a mechatronic CPM device, it is also necessary to consider the specific functions of the device itself, not just its mechanical parameters. Citing papers [3, 7], these functions boil down to:

- $\quad$ adjustability to a wide range of patient sizes;

- comfort, particularly for long uninterrupted application;

- use a variety of settings: while sleeping, while ambulating during the day, at home or in hospital;

- full anatomic range of motion capability and multi-axis motion capability;

- $\quad$ speed control and simple, straightforward operation;

- $\quad$ protection for unstable joints and protection for unstable joints;

- durability and lightweight;

- low cost simple solution.

In the original CPM design, it was assumed that it was to be a stationary solution, dedicated for use in hospitals and rehabilitation clinics. After the patient is trained properly, it will also be possible to use the system at home. Therefore, materials applied must be light, durable, and the technology is to be safe for the patient as well as for the personnel operating the CPM device. Coatings on structural elements should ensure easy cleaning and disinfection after treatment has been completed. The CPM design should withstand load under a patient body weight up to $120 \mathrm{~kg}$, thus it should have a $20 \%$ safety margin according to the weight of an adult male with $95 \mathrm{c}$ according to the anatomical atlas of human measures. Analogously, the global dimensions of the CPM mechanism's members relative to the length of part of the lower limb must ensure 
rehabilitation for the adult male population within the $5 \mathrm{c}$ to $95 \mathrm{c}$ range according to data from the atlas of human measures. The mechanism being the basic solution of the CPM device will be the double four-bar linkage and crank-slide mechanism described in [7].

The designed solution is to enable simultaneous rehabilitation of two limbs as well as each limb separately within the angle range of the knee's movement (from $-10^{\circ}$ to $+155^{\circ}$ ). A change of movement range does not require a change of the patient's position or additional activities related to finding a new, convenient position and re-fastening of limbs with stabilizing belts. Generally, in a typical seven-day period of rehabilitation following surgery, the value of the knee movement angle range is set, at maximum, on the CPM device from $105^{\circ}$ to $110^{\circ}$, while a range from $60^{\circ}$ to $70^{\circ}$ is used on the first day. Different parameters of the rehabilitation program can be set for each limb. The CPM device is to be equipped with a seat with an adjustable backrest for the patient undergoing rehabilitation. In addition, the CPM device is to enable adjustment of the position of the surface near the ankle on which the foot is to be supported. This relates to the angles of inclination of this surface relative to the knee flexion axis (significant when a spastic foot is being supported), as well as to a change of distance between the knee flexion axis to the foot support surface. The support in the thigh part of the limb must be adjustable for the purpose of achieving a better fit, however the main support in the shin part is not adjustable. In both the thigh and shin parts, as well as feet, the limb is to be fastened to the CPM device by means of velcro belts that are adjustable to the limb's size. An additional function of the CPM device concerns development of the design, which is to serve for simultaneous rehabilitation of the lower limb in both the knee and ankle joint.

A significant assumption of the design is the presence of a system that decouples the operation of the CPM drive mechanism, causing it to become "loose", i.e., enabling quick release of a limb if the patient suffers pain that they are not able to withstand. An improperly set up device, causing additional pain, may contribute to limitation of the mobility of the joint that was operated upon, extend hospitalization, and even deteriorate joint function after the completion of treatment. The system provides an additional protection against the risk of an unexpected error by the person operating the CPM device and will provide the capability of effective post-operative pain control as a result of objectivization of sensation as well as the capability of counteracting pain in the case of excessive intensity;.

The main drive of the CPM device should ensure fluid adjustment of rotational speed throughout the entire movement range (one period of full movement in initial passive rehabilitation may last up to 3 minutes). It is assumed that, under standard load, the CPM machine may operate up to 6500 hours (over 3 million cycles) over the course of one year. Most commercially available CPM machines are built for a service life of at least 5000 hours of use, and a few are serviceable up to 15,000 hours $[3,7]$. This arises from the fact that a CPM device is used in the very early stage of post-operative rehabilitation in medical practice, even two days after a surgical procedure, and is usually used between 7 and 10 days. In certain, individual cases, use of the device is extended even up to three weeks. The literature indicates that use of the device for more than 21 days from the time of surgery does not bring any additional, positive effects, and rehabilitation should be extended by other forms of kinesiotherapy with the goal of increasing movement range in a given joint (e.g. free active exercises, stretching, isotonic exercises, etc.) [4, 5].

\section{Description of the design of the hybrid dual-limb CPM rehabilitation machine}

An original hybrid CPM machine solution was designed as a result of an analysis of commercially available rehabilitation device solutions and identification of current rehabilitation needs at medical centers, with simultaneous consideration of own engineering assumptions. SolidWorks 2015/2016 CAD engineering software was used for this purpose. Figures 1 and 2 show the components of the conceptual solution of the rehabilitation system in the form of solid models. 


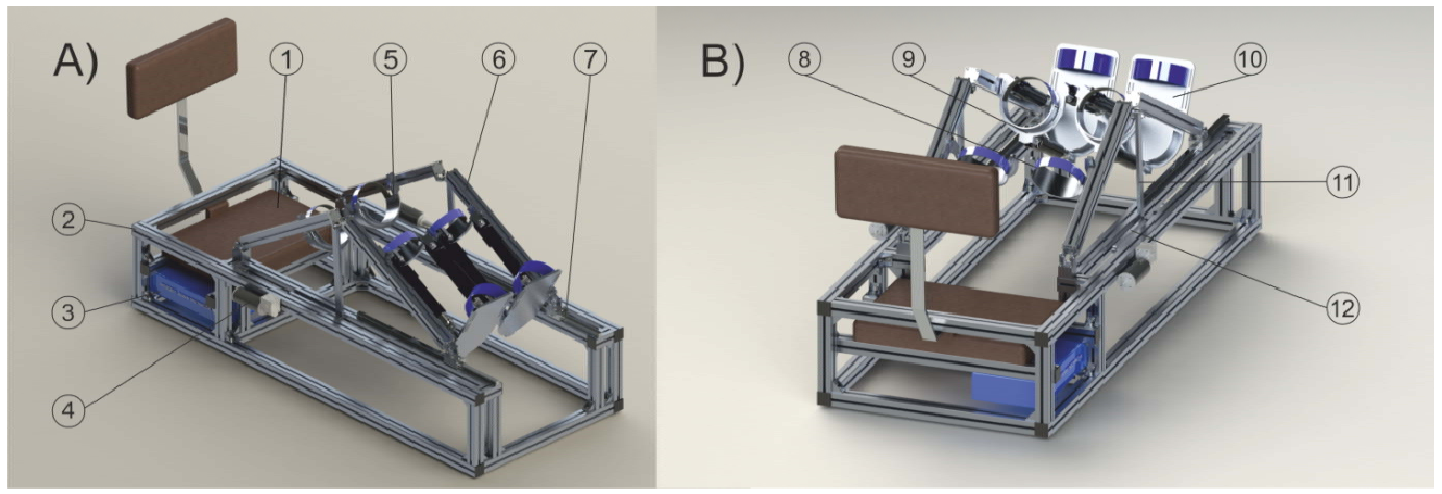

Fig.1. View of CPM device's computational SolidWorks CAD model A) front view, B) back view.

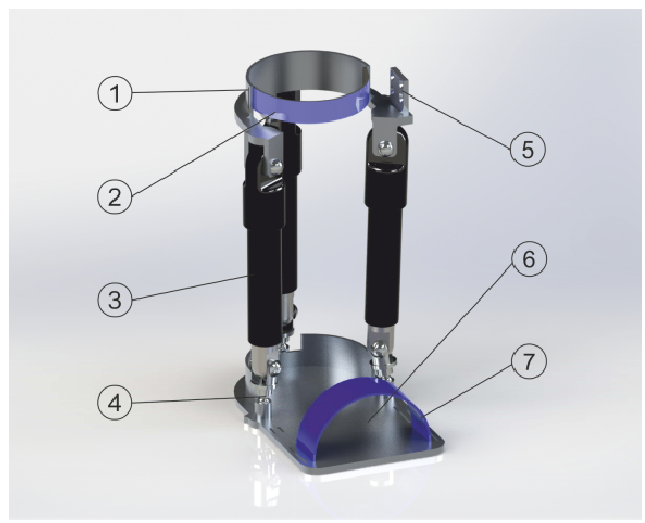

Fig.2. View of CPM device's Delta robot part computational SolidWorks model.

The design of the solution presented in Fig.1 is mainly based on a system of light anodized aluminum sections (EN AW-6061-T6 alloy), supplemented by joining and assembly elements. They were used to build a bolt-fastened frame (2) on which a fixed seat with adjustable backrest (1) was mounted, the height and position of which is adjusted depending on the patient's position, as well as active manipulator arms (6) for the left and right limb. A programmable microprocessor control system (3), which is responsible for controlling servomotors with encoders (4), is installed under the seat. The arms of active manipulators can move asynchronously (each of them may perform different rehabilitation programs) or synchronously according to set parameters, as needed. The members making up active manipulators are joined by specially designed hinge joints, which provide the full movement range of the knee (i.e., from $-10^{\circ}$ to $+155^{\circ}$ ) and are attached to the linear guide (7) fastened on the frame (2). Moving members (11) and (12) along with servomotor (4) make up the drive system of the main active arms mechanism (6). Fixed supports are attached to these arms under the thigh part of the limb (5) along with velcro belts (8) fastening the thigh for better limb restraint. The positions of these supports can be changed slightly be turning them to three positions (nearer to or farther from the axis of the joined arms' rotation). This will provide a better fit according to the needs and the length of the patient's limbs. A fixed support along with a fastening belt (9) is placed on the second manipulator arm and serves as the base for the Delta manipulator (10) in the shin and ankle part of the lower limb.

Figure 2 presents the details of the Delta manipulator's construction. New designations of elements have been adopted here. The base of the manipulator consists of the shin support (1) along with the velcro fastening strap (2) for better limb restraint. This support is fastened by a system of four bolts (5) to the active manipulator arm. The arms of the Delta manipulator are made up of electric linear actuators along with an encoder (3) as well as arms (4) forming rotating kinematic pairs with actuators, as well as spherical 
kinematic pairs with the foot support plate (6). An additional velcro strap (7) is fastened to the foot support plate (6) for the purpose of fastening the foot during rehabilitation exercises. The position of the support plate is adjusted by a unit of linear actuators with an encoder (3). This pertains to both the distance of the Delta manipulator from the base and angles between foot (ankle) position and the knee's axis of rotation. Parameters of the active manipulator's movement and of the position, and potentially movement parameters, of the Delta manipulator are set by means of an operator's touch screen attached to the hybrid dual-limb CPM rehabilitation machine.

\section{Computer analysis of displacements, speeds and accelerations of the designed CPM machine's mechanism}

SAM ver. 6.1 computer software was used to analyze the movement of the CPM machine's mechanism. The mechanism was modeled in this software according to the dimension of the virtual model in SolidWorks software (i.e., connections of members with a given length at specific angles relative to one another) for testing. In addition, the drive was added to the mechanism under the assumption that full rotation of the drive member lasts 15 seconds, and this movement will consist of 100 intervals. This assumption arises from limitations of knee joint movement after an arthroplasty procedure due to damaged tissue and pain. For the purpose of analysis, trajectories of the mechanism's points over the course of movement were investigated, a hodograph was created, and accelerations were determined at points of the mechanism corresponding to the axis of rotation of the knee undergoing rehabilitation as well as to the sliding axis corresponding to limb extension (Fig.3). The length and relative position of members 3 and 4 have a fundamental impact on the movement of the entire CPM mechanism and on the shape of the path and motion trajectory of points on the mechanism corresponding to the patient's knee movement. For the purposes of analysis, the lengths and relative positions of these members were changed appropriately in order to provide the greatest possible movement range in the knee joint with total rotation of the drive member (4). Speed and acceleration values were also considered, with an additional analysis of speed and acceleration hodographs, and absolute speed and acceleration values were evaluated (Fig.4).

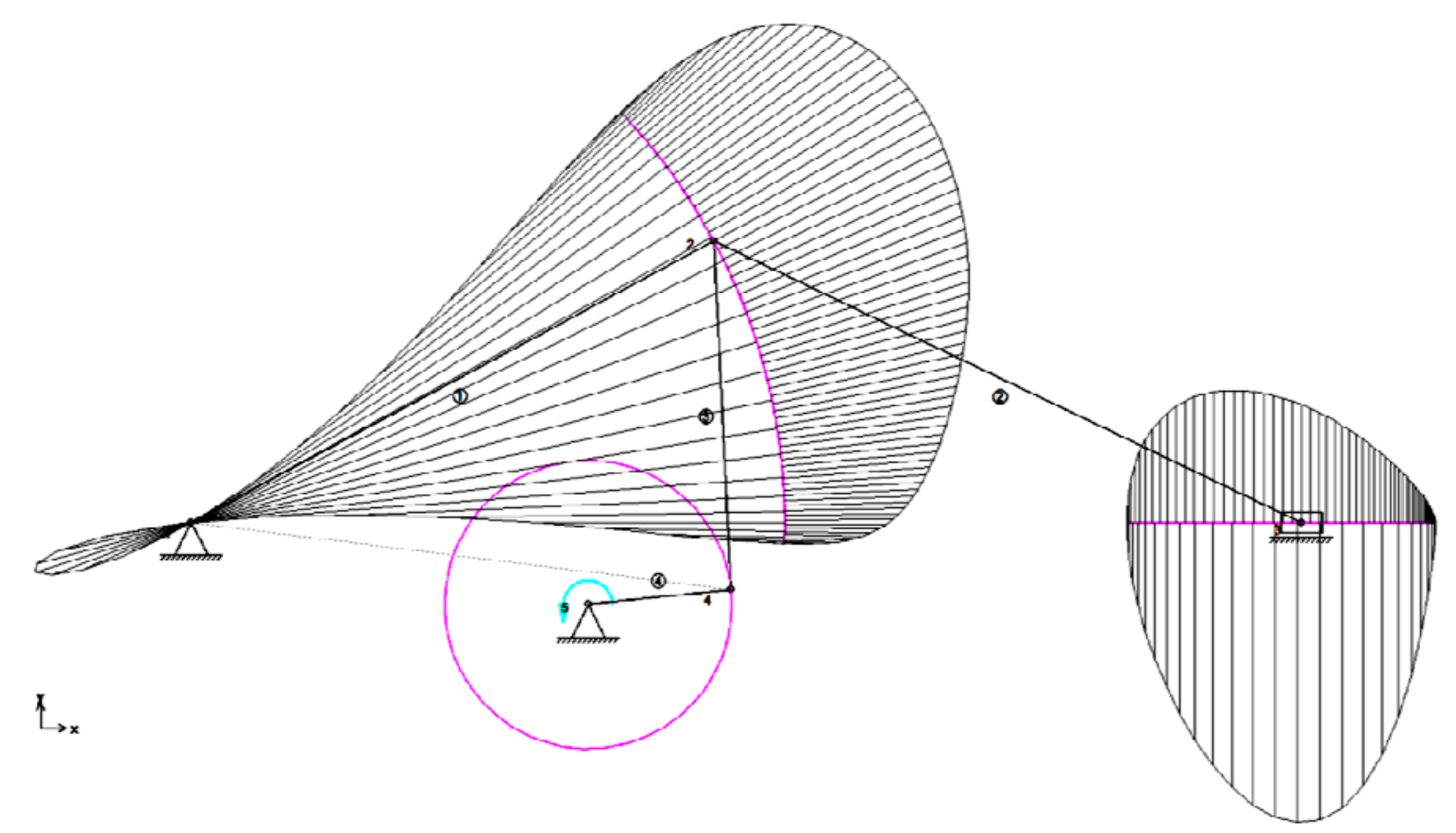

Fig.3. View of hodograph of velocity and acceleration of mechanism of CPM device created in SAM 6.1. 


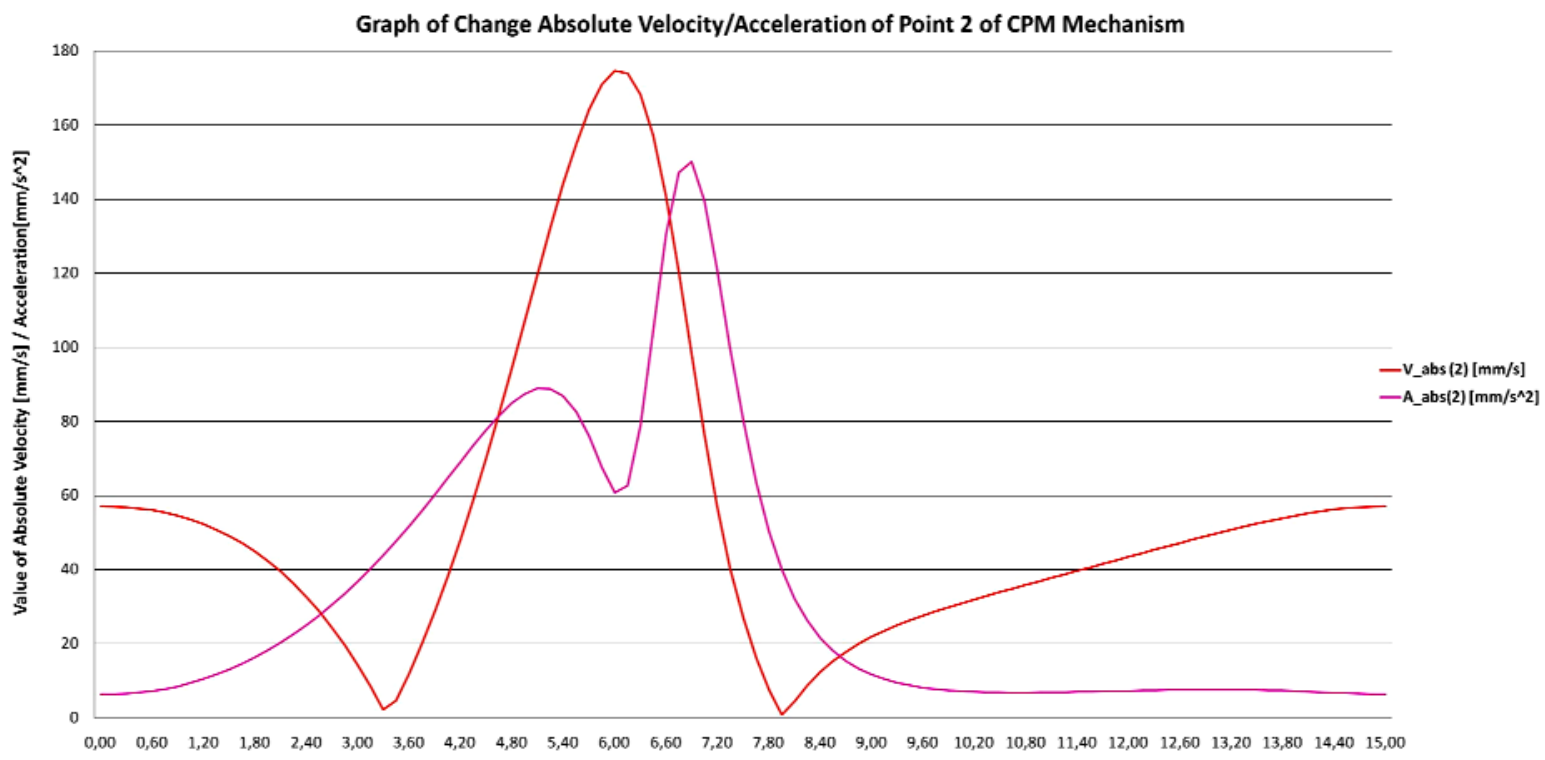

Fig.4. Graph of change in absolute velocity (red line) and acceleration (pink line) of point 2 of CPM mechanism created in SAM 6.1.

An analysis of the results shows that the greatest speed value $[174.58 \mathrm{~mm} / \mathrm{s}]$ was obtained in the 6 th second of movement of the drive member, and the greatest acceleration value $\left[150.01 \mathrm{~mm} / \mathrm{s}^{2}\right]$ was obtained in the 6.9th second of movement. As a result of analyses of different configurations (i.e., of the length and relative position of members 3 and 4, making up the CPM machine's drive mechanism), it was necessary to correct the lengths assumed initially. In the case of member 3 - from $310 \mathrm{~mm}$ to $299.5 \mathrm{~mm}$, and in the case of member 4 - from $210 \mathrm{~mm}$ to $123.4 \mathrm{~mm}$. This enabled the mechanism to operate properly according to previously made assumptions.

\section{Results of static finite element analysis in ANSYS of active arm of CPM device}

The solid model of the CPM device, created in SolidWorks 2015/2016, underwent static numerical analysis using the finite element method by means of ANSYS 13 software. Only the results obtained for the active arm are presented in this paper, and for the purposes of analysis, these results were appropriately prepared (all roundings and bevels were removed on modeled elements of the virtual model, joining elements in the design were removed) in order to speed up analysis and eliminate errors of superposition of the finite element mesh. The appropriate materials were also applied to specific parts of the model (data of materials used presented in Tab.1).

Table 1. Material properties used to CAE simulation.

\begin{tabular}{|l|l|l|}
\hline \multicolumn{1}{|c|}{ Property } & \multicolumn{1}{|c|}{ Stainless Steel } & Aluminium Alloy 6061 \\
\hline Density & $7750 \mathrm{~kg} \mathrm{~mm}^{\wedge}-3$ & $2770 \mathrm{~kg} \mathrm{~mm}^{\wedge}-3$ \\
\hline Compressive Yield Strength & $207 \mathrm{MPa}$ & $280 \mathrm{MPa}$ \\
\hline Tensile Yield Strenght & $207 \mathrm{MPa}$ & $280 \mathrm{MPa}$ \\
\hline Tensile Ultimate Strength & $586 \mathrm{MPa}$ & $310 \mathrm{MPa}$ \\
\hline Young's Modulus & $193 \mathrm{GPa}$ & $71 \mathrm{GPa}$ \\
\hline Poisson's Ratio & 0.31 & 0.33 \\
\hline Bulk Modulus & $169.3 \mathrm{GPa}$ & $69.608 \mathrm{GPa}$ \\
\hline Shear Modulus & $73.664 \mathrm{GPa}$ & $26.692 \mathrm{GPa}$ \\
\hline
\end{tabular}


The model prepared in this manner consisted of 22 solids in total, and its weight amounted to approx. $11.5 \mathrm{~kg}$ when material constants were accounted for. The proper joints of kinematic pairs were defined between the solids making up the numerical model by using fixed, rotating, sliding, cylindrical and spherical joints. Contacts between solids were created automatically when the arm's geometry was imported. The automatic finite element mesh creation method was used for the purpose of analysis, and only the size of the basic finite element was defined to be $7.5 \mathrm{~mm}$.

Figure 5 presents a view of the defined finite element mesh superimposed on the active manipulator's model, and detailed data concerning the model prepared for CAE analysis are given in Tab.2. The DCS Delta Coordinate System was additionally defined at the point of connection of the delta manipulator to the arm of the main active manipulator.

For the purposes of static analysis, the numerical model was loaded with torques with values of 20 and $-20 \mathrm{Nm}$ at the connection point between the drive and the driving member. The $20 \mathrm{Nm}$ value signifies operation of the drive during the extension phase of the limb undergoing rehabilitation (clockwise rotation of the member), and the $-20 \mathrm{Nm}$ value is the torque required to flex the limb undergoing rehabilitation (counterclockwise rotation of the member). In addition, forces of the following values were applied to the arm: 1) force of gravity; 2) $240 \mathrm{~N}$ force - load acting in the direction of gravity on the patient's thigh support; 3) $107 \mathrm{~N}$ force - load of the shin support acting in the direction of gravity; 4) $35 \mathrm{~N}$ force - load of foot on the support plate of Delta manipulator.

Table 2. CPM device properties - CAE analysis.

\begin{tabular}{|l|c|}
\hline \multicolumn{2}{|c|}{ CPM device properties - CAE analysis } \\
\hline Total Mass $[\mathrm{kg}]$ & 11.567 \\
\hline Mesh & Automatic Method - element size $7.5 \mathrm{~mm}$ \\
\hline Bodies & 22 \\
\hline Nodes & 172071 \\
\hline Elements & 53026 \\
\hline Contacts type & Bonded - automatic \\
\hline Joints revolte & Fixed, Revolte, Translational, Cylindrical, Spherical \\
\hline Material - Ansys Library & Stainless Steel, Aluminium Alloy 6061 \\
\hline Standard Earth Gravity $\left[\mathrm{mm} / \mathrm{s}^{2}\right]$ & 9806.6 \\
\hline Moment $[\mathrm{Nm}]$ & $20 ;-20$ \\
\hline Force Vector $[\mathrm{N}]$ & $240 ; 107 ; 35$ \\
\hline
\end{tabular}

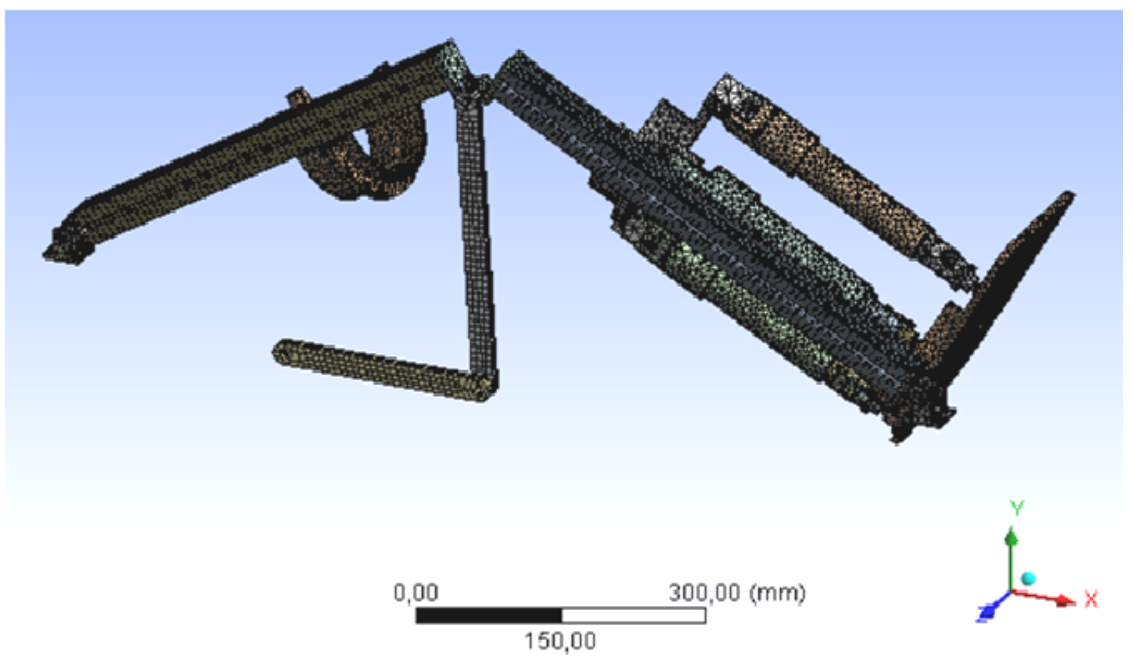

Fig.5. View of mesh use to ANSYS analysis of CPM device. 
The values of the aforementioned forces acting on the construction were determined according to an anatomical atlas of human measures for an adult male with $95 \mathrm{c}$, with a $20 \%$ safety margin.

The following were obtained as the results of analysis after the aforementioned parameters were set: Max Value of Total Deformation, Max Value of $X, Y, Z$ - Axis Directional Deformation in DCS - Delta Coordinate System, Max Value of Equivalent (von - Misses) Elastic Strain, Max Value of Equivalent (von Misses) Stress. The values of obtained the results of numerical simulations are given in Tab.3.

Table 3. Results of CAE simulations.

\begin{tabular}{|l|c|c|}
\hline Parameters Case of study & $\begin{array}{c}\text { Moment } \\
\text { Value +20Nm }\end{array}$ & $\begin{array}{c}\text { Moment } \\
\text { Value -20Nm }\end{array}$ \\
\hline Max value of Total Deformation [mm] & 2.082 & 3.0639 \\
\hline Max value of X - Axis Directional Deformation in DCS [mm] & 0.53618 & 0.81884 \\
\hline Max value of Y - Axis Directional Deformation in DCS [mm] & 0.04664 & 0.09049 \\
\hline Max value of Z - Axis Directional Deformation in DCS [mm] & 0.45560 & 0.45038 \\
\hline Max value of Equivalent (von - Misses) Elastic Strain & 0.0013 & 0.0013 \\
\hline Max value of Equivalent (von - Misses) Stress [MPa] & 148.81 & 196.58 \\
\hline
\end{tabular}

Figures 6 and 7 present results from ANSYS software graphically for a less favorable case, i.e., when the limb undergoing rehabilitation is extended and the drive (torque) moves clockwise along with driving members. Figures 6 and 7 present the CPM device in deformed and non-deformed state when the automatic scale factor is set and show the points at which values reach their maximum and minimum.
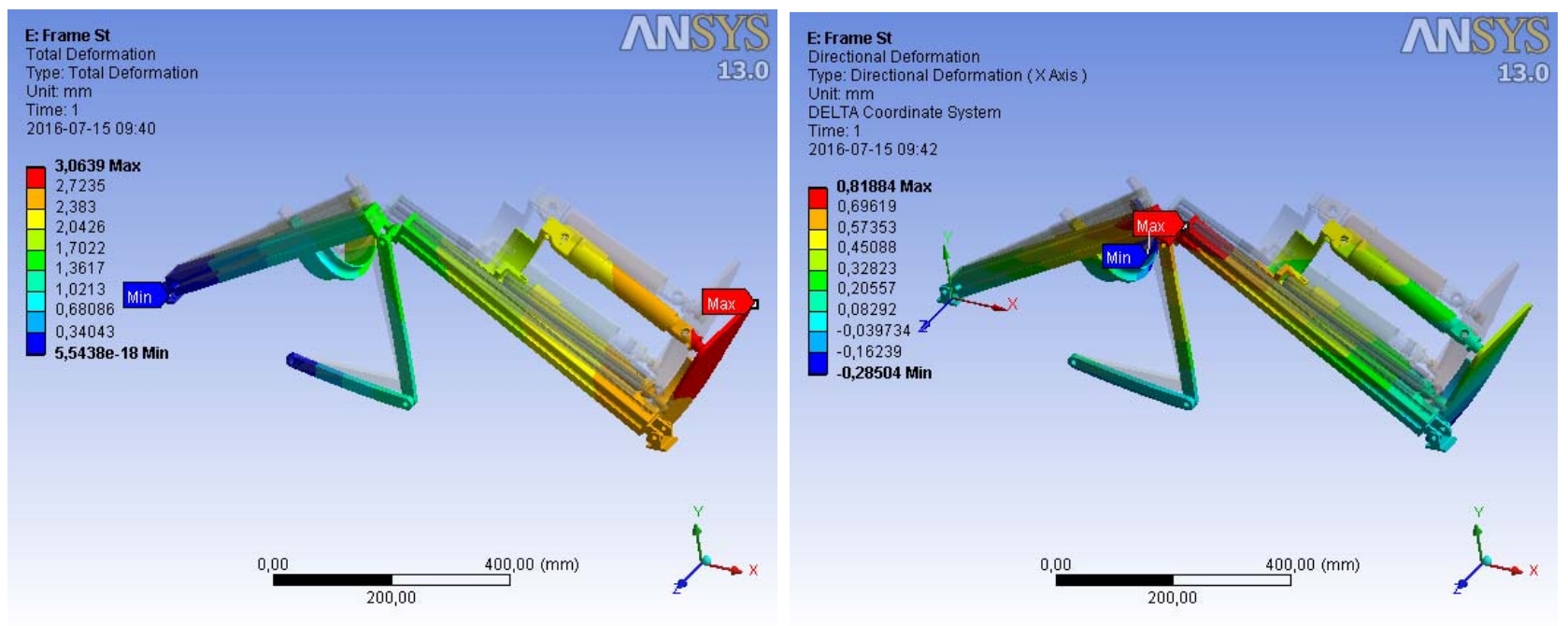

Fig.6. Results of ANSYS analysis of CPM device: 1) max value of total deformation of active manipulator in case of study $-20 \mathrm{Nm}$. 2) max value of directional deformation in $X$-Axis in case of study $-20 \mathrm{Nm}$.

The results of the numerical simulation unequivocally show that the greatest value for the total deformation test amounts to $3 \mathrm{~mm}$ for the two tested cases, when the torque assumes the value of $-20 \mathrm{Nm}$. However, the maximum value pertains to the foot support element, as shown in Fig.6. 


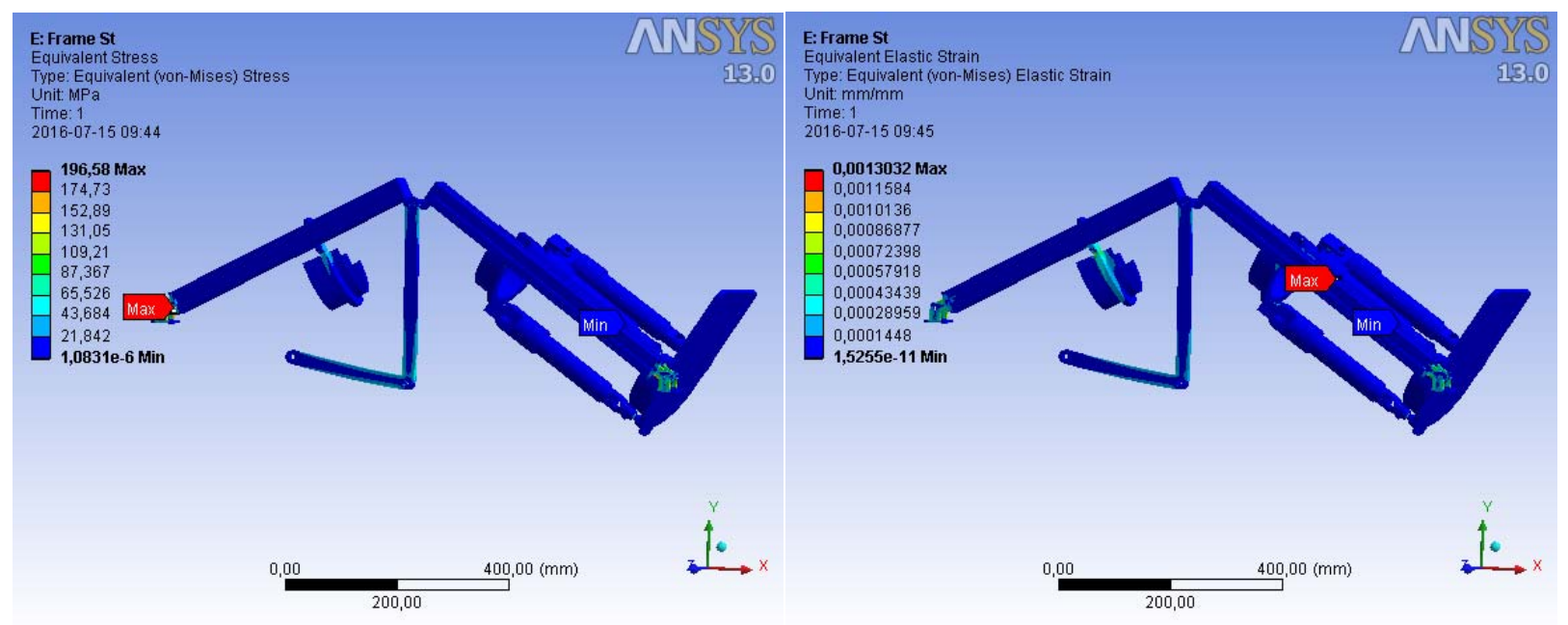

Fig.7. Results of ANSYS analysis of CPM device in case of study -20Nm: 1) equivalent (von-Mises) stress, 2) equivalent (von-Mises) elastic strain.

In practice, this value can be reduced by selecting a different material for the shin support or by changing the geometry of the support itself. On the knee joint axis, however, this value is just approx. $1 \mathrm{~mm}$, which does not have a significant influence on the quality of the process of knee joint rehabilitation. In the case of the directional deformation test, the highest value was obtained in the case of deformation on the $\mathrm{X}$ axis (Fig.6 case 2). This value amounts to approx. $0.8 \mathrm{~mm}$. In the practical solution of the CPM device, this value does not have a negative impact on the quality of movement in the knee joint. However, it should be remembered that results pertain to patients with a body weight of $120 \mathrm{~kg}$, and the given values will be less for a person with a lower weight.

In addition, numerical simulations were carried out with the purpose of indicating the point of maximum stresses in the CPM device construction according to the Huber-Mises hypothesis. Results are represented graphically in Fig.7. Results obtained in the case of the equivalent (von - Misses) stress test confirm the authors' previous conjectures as to the point where maximum stresses occur in the construction of the CPM device. Their value (approx. $197 \mathrm{MPa}$ ) is not high, however this suggests that the given hinge joint - the fixed support of the active main arm - should be reinforced in the actual construction. Equivalent (von - Misses) elastic strain tests show that the greatest values of elastic strain occur, as expected, at the fastening point of the shin support. However, the maximum elastic strain value is low enough (equal to 0.0013) that it has no significant influence on the behavior of the CPM device in static load states.

\section{Conclusions}

The goal of rehabilitation after total knee arthroplasty is to restore the range of motion (ROM) in the knee joint that was operated onto the greatest possible extent $[3,5,7]$ as well as full muscular control over this joint. Different forms of physiotherapy are applied in post-operative rehabilitation, including different types of kinesiotherapy supported, in a great degree, by passive exercises using CPM devices. Original research, preceded by many analyses of commercial solutions and analyses of the literature in the field of CPM rehabilitation devices, resulted in the development of an original design of a hybrid, dual-limb CPM rehabilitation device. The conceptual model of the CPM device presented in this paper responds to the current needs of patients and medical centers concerned with rehabilitation after total knee arthroplasty procedures by virtue of the engineering solutions applied. Thanks to the relatively easy capability of adjusting the device to the dimensions of different patients' limbs, as well as the capability of setting different exercise programs separately for each limb, functionality was expanded in comparison to CPM systems currently available on the market. A significant factor decisive to the solution's innovation is the 
application of an additional Delta manipulator, thanks to which persons with spastic feet and differing limb lengths can undergo rehabilitation. Another advantage of the solution is the capability of simultaneous rehabilitation in the area of the ankle joint, besides knee rehabilitation following arthroplasty, which significantly expands the range of kinesiotherapeutic exercises.

The use of results of analyses from CAD and CAE systems, particularly engineering packages utilizing finite element methods, allows a quicker and easier optimization of the kinematic and mechanical functions of new technical solutions. This gives the designer a better understanding of operation and confirms the design assumptions adopted. The results of static analyses will also be used to introduce correction coefficients into the control system in order to achieve qualitative positioning of the biomedical construction.

Thanks to the application of SAM 6.1 software, analysis and optimization of a flat mechanism can be carried out very quickly using tools and knowledge from the field of machine theory without engaging significant funds and computing power of computers used for modeling. Based on the results of analysis, the appropriate corrections have been made to the sizes of characteristic members making up the mechanism, with consideration of absolute trajectories, speeds and accelerations.

In future work on the design of the mechatronic CPM device, it is planned to use Software in the Loop Simulation (SiLS) and Hardware in the Loop Simulation (HiLS) simulation techniques in order to prototype and optimize the parameters of the device's controller, as well as to build a laboratory demo which will be tested on patients at a rehabilitation center after a testing period.

This work was performed within the framework of statutory work of the Department of Automatic Control and Robotics at Bialystok University of Technology no. S/WM/1/2016 and financed with funds for scientific research from the Ministry of Science and Higher Education.

\section{References}

[1] Markuszewski J., Kokoszka P. and Ogrodowicz P. (2015): Arthroplasty of joints. - In: Wiktor's Dega orthopedics and rehabilitation, (Kruczyński J. and Szulc A., Ed.). - Warsaw: PZWL (in Polish).

[2] Kurtz S., Ong K., Lau E., Mowat F. and Halpern M. (2007): Projections of primary and revision hip and knee arthroplasty in the United States from 2005 to 2030. - Journal of Bone and Joint Surgery Am, 89.4, pp.780-785.

[3] Saringer J. (1993): Engineering aspect of the design and construction of continuous passive motion device for humans. - In: Continuous Passive Motion: A Biological Concept for the Healing and Regeneration of Articular Cartilage, Ligaments, and Tendons: From Origination to Research to Clinical. Baltimore (Salter R. Ed.) Williams\&Wilkins.

[4] Driscoll O., Shawn W. and Giori N.J. (2000): Continuous passive motion (CPM): theory and principles of clinical application. - Journal of rehabilitation research and development, 37.2, pp.179-188.

[5] Yashar A.A., Venn-Watson E., Welsh T., Colwel C.W. Jr. and Lotke P. (1997): Continuous passive motion with accelerated flexion after total knee arthroplasty. - Clinical Orthopaedics and Related Research, 345, pp.38-43.

[6] Widuchowski J., Kusz D., Widuchowski W. and Pierzchała A. (2004): Total joint replacement - the operating method for the treatment of advanced disease and distortion of the knee. - Fizjoterapia Polska, vol.4, No.4, pp.403-410 (in Polish).

[7] Trochimczuk R. and Kuźmierowski T. (2014): Kinematic analysis of CPM machine supporting to rehabilitation process after surgical knee arthroscopy and arthroplasty. - International Journal of Applied Mechanics and Engineering, vol.19, No.4, pp.841-848.

Received: October 21, 2016

Revised: November 30, 2016 